\title{
A GLOBAL VLBI/LLR ANALYSIS FOR THE DETERMINATION OF PRECESSION AND NUTATION CONSTANTS
}

\author{
P. CHARLOT ${ }^{1,2}$, O. J. SOVERS ${ }^{1}$, J. G. WILLIAMS ${ }^{1}, X_{\text {X NEWHALL }}^{1}$ \\ ${ }^{1}$ Jet Propulsion Laboratory, California Institute of Technology \\ 4800 Oak Grove Drive, Pasadena, California 91109 \\ ${ }^{2}$ Central Bureau of IERS, Observatoire de Paris \\ 61 Avenue de l'Observatoire, 75014 Paris, France
}

\begin{abstract}
Two decades of LLR data and one decade of VLBI data are combined in a global analysis to yield improved estimates of the Earth's precession and nutation. In this analysis, LLR provides a strong determination of precession, while VLBI is stronger in fixing nutation terms with short periods. In all, 24 nutation amplitudes are estimated. The largest correlation coefficient, between precession and $18.6 \mathrm{yr}$ out-of-phase nutation in longitude, is 0.88 . With the exception of some $9 \mathrm{yr}$ and $18.6 \mathrm{yr}$ terms, formal uncertainties are 0.1 to 0.2 milliarcseconds.
\end{abstract}

\section{Introduction}

In the past five years, it has become obvious that the current precession and nutation theory recommended by the IAU is not adequate for reduction of modern astrometric observations, such as those acquired by the VLBI and LLR techniques. Discrepancies at the level of a few milliarcseconds, especially for the precession constant and $18.6 \mathrm{yr}, 1 \mathrm{yr}$, $0.5 \mathrm{yr}$ and 14 day nutation terms, have been revealed by VLBI and LLR observations (e.g. Herring et al., 1986; Williams et al., 1990). It is of great importance to establish more accurate precession and nutation constants in order to improve VLBI and LLR analyses for astrometry and geodesy. However, based on theoretical considerations, any improvement of the quality of these constants is presently difficult because geophysical properties of the Earth's interior (e.g. core flattening, mantle inelasticity) are not known well enough. The only way to obtain more accurate precession and nutation constants is to estimate them from the VLBI and/or LLR observations themselves. Comparing precession and nutation constants estimated from LLR and Deep Space Network VLBI data (see Section 4) shows that estimates of the short-period nutation terms ( $1 \mathrm{yr}, 0.5 \mathrm{yr}, 14$ day) are more accurate when derived from VLBI data, while estimates of the precession constant and the longperiod nutation terms $(18.6 \mathrm{yr}, 9 \mathrm{yr})$ in longitude are more accurate when derived from LLR data. Therefore, combining VLBI and LLR data is the best way to improve the estimation of precession and nutation constants with the present data, because it takes advantage of the unique and complementary strengths of the two techniques.

For the purpose of estimating precession and nutation constants, we have developed a global analysis of VLBI and LLR observations. This global analysis (described in Section 2) is performed by combining the VLBI and LLR information matrices derived from 
the data equations with the VLBI and LLR software of the Jet Propulsion Laboratory (JPL). We emphasize that, in this analysis, the full VLBI and LLR information matrices are retained to produce combined VLBI/LLR estimates of the precession constant and 24 nutation amplitudes. Section 3 describes the data sets and modeling used in our analysis, while Section 4 presents and discusses our results, including a comparison between the VLBI, LLR, and VLBI/LLR estimates and correlation coefficients.

\section{The Algorithm Used to Combine VLBI and LLR Data}

In the past decade, VLBI software (MODEST) (Sovers and Fanselow, 1987) and LLR software (LPRED) has been developed at JPL. Both of these programs use the Square Root Information Filter (SRIF) algorithm, based on repeated Householder transformations, to triangularize the data equation matrix. This algorithm is extensively described in Bierman (1977). One advantage of the SRIF algorithm is that it allows one to add new data equations to a previously analyzed set of data equations and produce "updated" parameter estimates without reanalyzing all the data. In practice, this saves much computer time when large amounts of data are to be processed and updated. In our analysis, this capability has been used to combine observations of different types (VLBI and LLR).

The main steps of our joint VLBI/LLR analysis are shown in Figure 1. First, theoretical values of LLR ranges and VLBI delays and delay rates are calculated and differenced with observations to produce O-C's. This step is performed separately for VLBI and LLR measurements by using MODEST and LPRED. Then, the SRIF algorithm is applied to LLR O-C's and the R-matrix is retained before the calculation of the LLR parameter estimates. This matrix is an upper triangular matrix obtained by applying Householder transformations to the data equations (see Bierman, 1977). It is further used as an a priori information matrix in a way that mimics a VLBI R-matrix, when the SRIF algorithm is applied to VLBI O-C's. This latter step requires identification of parameters common to VLBI and LLR and matching their names and units in order to produce consistent joint VLBI/LLR parameter estimates. It is to be noted that for our analysis, only precession and nutation constants have been considered as common VLBI/LLR parameters.

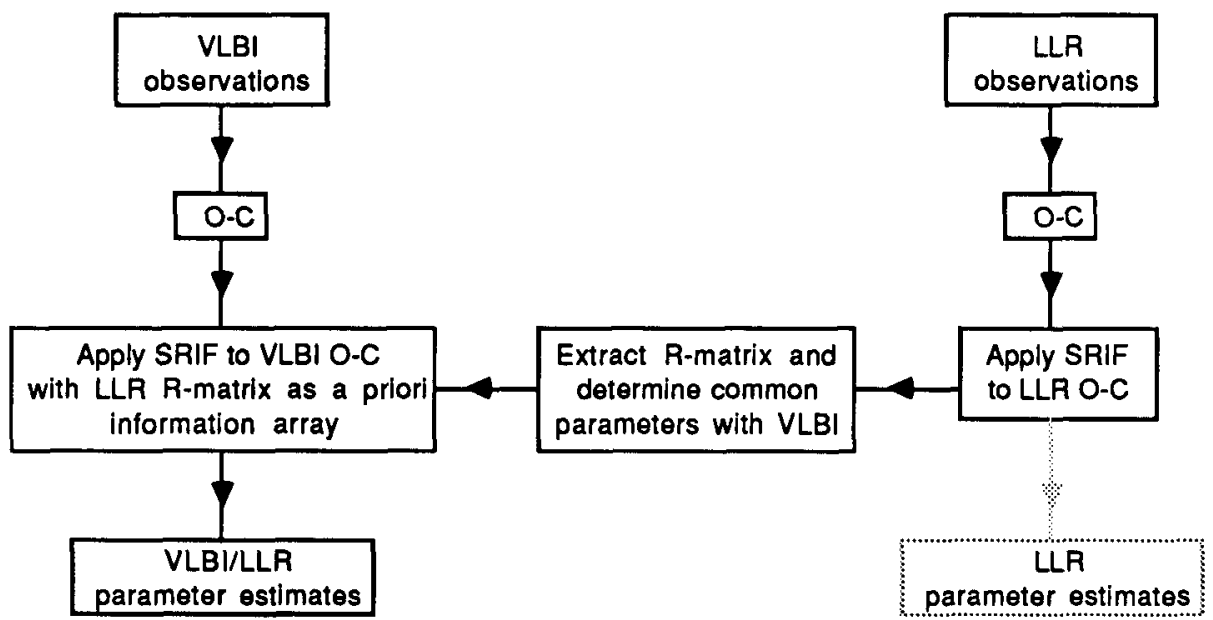

Figure 1: Main steps of the joint VLBI/LLR analysis. 


\section{Observations and Modeling}

Our VLBI data set consists of 68 dual-frequency (2.3 and $8.4 \mathrm{GHz})$ VLBI observing sessions carried out by the Deep Space Network (DSN) on two intercontinental baselines (Goldstone-Madrid and Goldstone-Tidbinbilla) between October 1978 and October 1989 . It is basically the same data set described by Sovers (1990), and includes 8578 dualfrequency Mark II delay and delay rate pairs and 423 Mark III pairs. The LLR data (described in more details by Williams et al., 1990) consist of 6293 ranges (distance measurements) from telescopes on the Earth to reflectors on the Moon acquired between August 1969 and December 1989. VLBI and LLR modeling was performed in Solar System Barycentric coordinates in terms of the mean equator and equinox of J2000.0, and largely adhered to the IERS standards (1989) for astronomical constants. The LLR analysis is based on the DE303/LE303 planetary and lunar ephemeris. Earth orientation parameters were adopted from a combination-of-techniques series obtained by Kalman filtering (Gross 1990, private communication). More information about VLBI and LLR modeling is found in Sovers (1990) and Williams et al. (1990), respectively.

In our analysis, the precession constant and both the in-phase and out-of-phase nutation terms of periods $18.6 \mathrm{yr}, 9 \mathrm{yr}, 1 \mathrm{yr}, 0.5 \mathrm{yr}, 14$ days, and 433.2 days (retrograde free core nutation with reference epoch J2000) have been estimated. We emphasize that this is the first unconstrained analysis with the precession constant and the $18.6 \mathrm{yr}$ and $9 \mathrm{yr}$ nutation terms estimated together in the same fit. Such unconstrained analysis is possible because of the time span of our data, especially the LLR data which cover 20 years.

\section{Results}

Table 1 shows our combined VLBI/LLR estimates of 25 precession and nutation constants together with the individual VLBI and LLR estimates of these constants. The values indicated in this table are the corrections to the IAU nutation coefficients; all errors quoted in this paper are formal $1 \sigma$ uncertainties. Units are milliarcseconds (mas) for nutation, and mas/yr for precession. The nutation terms are labeled with subscripts corresponding to term numbers in the 1980 IAU series, while the superscripts indicate the phase (sine or cosine).

Comparing the formal uncertainties of the individual VLBI and LLR estimates shows that LLR data provide more accurate estimates of the precession constant and the $18.6 \mathrm{yr}$ and $9 \mathrm{yr}$ nutation terms in longitude, while VLBI data provide more accurate estimates of the other nutation terms, especially the short-period nutation terms $(1 \mathrm{yr}, 0.5 \mathrm{yr}, 14-$ day). Notably, the formal uncertainty of the precession constant is smaller by an order of magnitude when derived from LLR data $(0.17$ mas $/ y r)$ than when derived from VLBI data $(1.87 \mathrm{mas} / \mathrm{yr})$. Estimates of the precession constant and the $18.6 \mathrm{yr}$ and $9 \mathrm{yr}$ nutation terms in longitude based on VLBI data have large uncertainties caused by extremely high correlation coefficients between these parameters as shown in Table 2 . In this table, only correlations exceeding 0.5 are printed; within each triplet, the order (top to bottom) is LLR, VLBI, and VLBI/LLR. For example, the correlation coefficient between the precession constant and the 18.6 yr out-of-phase nutation in longitude $\left(\Delta \psi_{1}^{c}\right)$ is 0.997 in the VLBI analysis, while it is only 0.64 in the LLR analysis. Such a huge correlation coefficient is explained by the shape of $\Delta \psi_{1}^{c}$, which is almost linear over the period of our VLBI observations (see Figure 2). Besides this correlation coefficient, 14 other correlation coefficients are larger than 0.50 in the VLBI analysis, 4 of them larger than 0.90 . By contrast, only 3 correlation coefficients are larger than 0.50 (maximum value of 0.64 ) in the LLR analysis. 
Table 1. Comparison of the VLBI, LLR, and Combined VLBI/LLR Estimates of 25 Precession and Nutation Constants

\begin{tabular}{|c|c|c|c|}
\hline & LLR & VLBI & VLBI/LLR \\
\hline Precession & $-2.66 \pm 0.17$ & $-5.17 \pm 1.87$ & $-2.83 \pm 0.15$ \\
\hline 18.6 yr $\Delta \varepsilon_{1}^{c}$ & $0.47 \pm 0.59$ & $1.57 \pm 0.26$ & $1.44 \pm 0.20$ \\
\hline$\Delta \psi_{1}^{*}$ & $-7.60 \pm 1.49$ & $-10.21 \pm 3.62$ & $-6.07 \pm 0.85$ \\
\hline$\Delta \varepsilon_{1}^{s}$ & $2.46 \pm 0.74$ & $2.80 \pm 0.54$ & $2.28 \pm 0.37$ \\
\hline$\Delta \psi_{1}^{c}$ & $3.12 \pm 1.52$ & $12.52 \pm 8.29$ & $3.05 \pm 0.96$ \\
\hline $9 \mathrm{yr} \quad \Delta \varepsilon_{2}^{c}$ & $-0.43 \pm 0.49$ & $0.18 \pm 0.23$ & $-0.12 \pm 0.18$ \\
\hline$\Delta \psi_{2}^{B}$ & $-0.22 \pm 1.04$ & $-0.88 \pm 1.76$ & $1.17 \pm 0.62$ \\
\hline$\Delta \varepsilon_{2}^{B}$ & $-0.29 \pm 0.50$ & $-0.17 \pm 0.34$ & $0.04 \pm 0.24$ \\
\hline$\Delta \psi_{2}^{c}$ & $1.09 \pm 1.02$ & $-1.24 \pm 1.56$ & $0.08 \pm 0.41$ \\
\hline $1 \mathrm{yr} \quad \Delta \varepsilon_{10}^{\mathrm{c}}$ & $1.59 \pm 0.34$ & $1.60 \pm 0.13$ & $1.69 \pm 0.11$ \\
\hline$\Delta \psi_{10}^{b}$ & $5.03 \pm 0.66$ & $4.26 \pm 0.34$ & $4.25 \pm 0.27$ \\
\hline$\Delta \varepsilon_{10}^{8}$ & $-1.87 \pm 0.27$ & $-0.25 \pm 0.12$ & $-0.54 \pm 0.10$ \\
\hline$\Delta \psi_{10}^{c}$ & $-0.39 \pm 0.47$ & $0.85 \pm 0.33$ & $0.81 \pm 0.25$ \\
\hline 0.5 yr $\Delta \varepsilon_{g}^{c}$ & $-0.65 \pm 0.27$ & $-0.58 \pm 0.11$ & $-0.50 \pm 0.10$ \\
\hline$\Delta \psi_{9}^{8}$ & $1.09 \pm 0.50$ & $1.70 \pm 0.33$ & $1.37 \pm 0.25$ \\
\hline$\Delta \varepsilon_{9}^{\Delta}$ & $1.02 \pm 0.29$ & $-0.61 \pm 0.12$ & $-0.44 \pm 0.10$ \\
\hline$\Delta \psi_{9}^{c}$ & $-0.02 \pm 0.52$ & $-0.28 \pm 0.30$ & $-0.27 \pm 0.24$ \\
\hline 14 day $\Delta \varepsilon_{31}^{c}$ & $0.80 \pm 0.47$ & $0.18 \pm 0.11$ & $0.07 \pm 0.10$ \\
\hline$\Delta \psi_{31}^{s}$ & $-2.97 \pm 0.93$ & $-0.49 \pm 0.30$ & $-0.74 \pm 0.23$ \\
\hline$\Delta \varepsilon_{31}^{6}$ & $0.91 \pm 0.42$ & $0.05 \pm 0.12$ & $-0.12 \pm 0.10$ \\
\hline$\Delta \psi_{31}^{c}$ & $0.75 \pm 0.82$ & $0.68 \pm 0.31$ & $0.56 \pm 0.25$ \\
\hline $\mathrm{FCN} \quad \Delta \varepsilon_{F}^{c}$ & $-0.12 \pm 0.26$ & $-0.29 \pm 0.13$ & $-0.34 \pm 0.11$ \\
\hline$\Delta \psi_{F}^{s}$ & $-0.71 \pm 0.42$ & $1.21 \pm 0.42$ & $0.04 \pm 0.28$ \\
\hline$\Delta \varepsilon_{F}^{b}$ & $-1.03 \pm 0.25$ & $0.10 \pm 0.15$ & $-0.13 \pm 0.13$ \\
\hline$\Delta \psi_{F}^{c}$ & $0.19 \pm 0.50$ & $-0.93 \pm 0.35$ & $-0.61 \pm 0.26$ \\
\hline
\end{tabular}

LLR data cover a period long enough to properly separate the precession constant and the $18.6 \mathrm{yr}$ and $9 \mathrm{yr}$ nutation terms.

In the combined VLBI/LLR analysis, correlation coefficients are significantly reduced relative to those of the VLBI analysis alone: only 8 correlation coefficients are larger than 0.50 (instead of 15 in the VLBI analysis), and the maximum value is 0.88 (instead of 0.997 in the VLBI analysis). It is also to be noted that formal uncertainties of parameters are 
Table 2. Comparison of Correlation Coefficients $|\rho|>0.5$ for the Precession Constant and the $18.6 \mathrm{yr}$ and $9 \mathrm{yr}$ Nutation Terms.

\begin{tabular}{|c|c|c|c|c|c|c|c|c|}
\hline & $\Delta \varepsilon_{1}^{c}$ & $\Delta \psi_{1}^{s}$ & $\Delta \varepsilon_{1}^{g}$ & $\Delta \psi_{1}^{c}$ & $\Delta \varepsilon_{2}^{c}$ & $\Delta \psi_{2}^{s}$ & $\Delta \varepsilon_{2}^{s}$ & $\Delta \psi_{2}^{c}$ \\
\hline Prec. & $\begin{array}{l}\ldots \\
\ldots \\
\ldots\end{array}$ & $\begin{array}{r}\ldots \\
0.91 \\
\ldots\end{array}$ & $\begin{array}{l}\ldots \\
\ldots \\
\ldots\end{array}$ & $\begin{array}{c}0.64 \\
0.997 \\
0.88\end{array}$ & $\begin{array}{l}\ldots \\
\ldots \\
\ldots\end{array}$ & $\begin{array}{c}\ldots \\
0.86 \\
0.52\end{array}$ & $\begin{array}{l}\cdots \\
\ldots \\
\ldots\end{array}$ & $\begin{array}{r}\ldots \\
0.91 \\
\ldots\end{array}$ \\
\hline$\Delta \varepsilon_{1}^{c}$ & & $\begin{array}{l}\ldots \\
\ldots \\
\ldots\end{array}$ & $\begin{array}{c}\ldots \\
0.77 \\
0.66\end{array}$ & $\begin{array}{l}\cdots \\
\cdots \\
\ldots\end{array}$ & $\begin{array}{l}\ldots \\
\ldots \\
\ldots\end{array}$ & $\begin{array}{l}\ldots \\
\ldots \\
\ldots\end{array}$ & $\begin{array}{r}\ldots \\
0.74 \\
0.72\end{array}$ & $\begin{array}{l}\ldots \\
\ldots \\
\ldots\end{array}$ \\
\hline$\Delta \psi_{1}^{s}$ & & & $\begin{array}{l}\ldots \\
\ldots \\
\ldots\end{array}$ & $\begin{array}{r}\ldots \\
0.89 \\
\ldots\end{array}$ & $\begin{array}{l}\ldots \\
\ldots \\
\ldots\end{array}$ & $\begin{array}{l}0.57 \\
0.60 \\
0.72\end{array}$ & $\begin{array}{l}\ldots \\
\ldots \\
\ldots\end{array}$ & $\begin{array}{r}\ldots \\
0.97 \\
\ldots\end{array}$ \\
\hline$\Delta \varepsilon_{1}^{s}$ & & & & $\begin{array}{l}\ldots \\
\ldots \\
\ldots\end{array}$ & $\begin{array}{r}\ldots \\
0.82 \\
0.74\end{array}$ & $\begin{array}{l}\ldots \\
\ldots \\
\ldots\end{array}$ & $\begin{array}{l}0.58 \\
0.89 \\
0.82\end{array}$ & $\begin{array}{l}\ldots \\
\ldots \\
\ldots\end{array}$ \\
\hline$\Delta \psi_{1}^{c}$ & & & & & $\begin{array}{l}\ldots \\
\ldots \\
\ldots\end{array}$ & $\begin{array}{r}\ldots \\
0.88 \\
0.69\end{array}$ & $\begin{array}{l}\ldots \\
\ldots \\
\ldots\end{array}$ & $\begin{array}{r}\ldots \\
0.90 \\
\ldots\end{array}$ \\
\hline$\Delta \varepsilon_{2}^{c}$ & & & & & & $\begin{array}{l}\ldots \\
\ldots \\
\ldots\end{array}$ & $\begin{array}{r}\ldots \\
0.64 \\
\ldots\end{array}$ & $\begin{array}{l}\cdots \\
\cdots \\
\ldots\end{array}$ \\
\hline$\Delta \psi_{2}^{s}$ & & & & & & & $\begin{array}{l}\ldots \\
\ldots \\
\ldots\end{array}$ & $\begin{array}{r}\ldots \\
0.65 \\
\ldots\end{array}$ \\
\hline
\end{tabular}

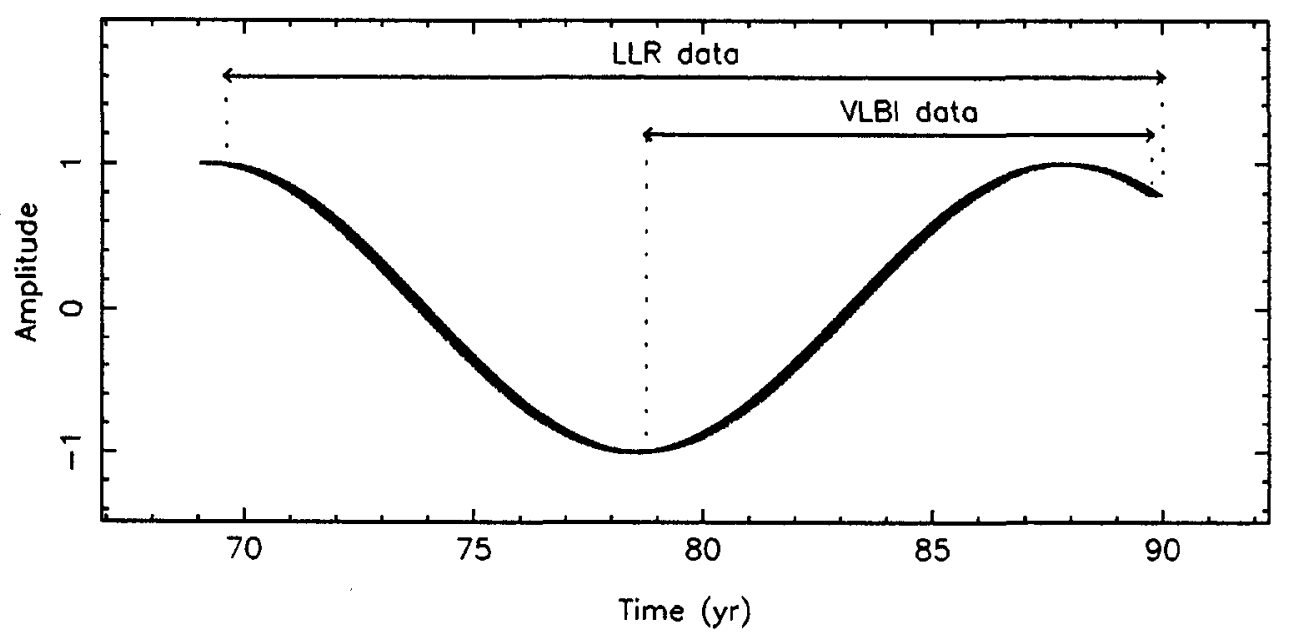

Figure 2: Shape of $\Delta \psi_{1}^{c}$ and coverage of VLBI and LLR data between 1969 and 1990. 
smaller in the combined analysis than in the individual VLBI and LLR analyses, with improvements of up to a factor of 2.5 (case of $\Delta \psi_{2}^{c}$ ). Based on the decrease of the correlation coefficients and the improvement of formal uncertainties, we argue that the combined VLBI/LLR analysis is stronger than either analysis alone. Comparing our VLBI/LLR estimates with the independent VLBI results of Herring (1988) and Zhu et al. (1990) indicates good overall agreement, with most of the differences at the $1-2 \sigma$ level. Because of the addition of the LLR data, our estimates of the precession constant and the $18.6 \mathrm{yr}$ nutation terms are stronger than those of Herring (1988) and Zhu et al. (1990). It is also worth noting that for the $9 \mathrm{yr}$ nutation terms, not previously estimated from VLBI data, our estimates of the corrections $(-0.12 \pm 0.18,1.17 \pm 0.62,0.04 \pm 0.24$, $0.08 \pm 0.41$ mas) are in good agreement with those from the deformable-Earth series of Zhu et al. (1990) $(-0.25,1.23,0.0,0.0$ mas $)$.

\section{Conclusion}

25 precession and nutation parameters are estimated in a combined analysis of 20 years of LLR data and 11 years of VLBI data. This global VLBI/LLR analysis combines the information matrices derived separately from the VLBI and LLR data equations. Comparisons of formal uncertainties and correlation coefficients between various precession and nutation parameters show that the combined VLBI/LLR analysis is stronger than either the VLBI or the LLR analysis alone. This combined analysis provides estimates of the precession constant and the $18.6 \mathrm{yr}$ nutation terms that are stronger than any previous results. It also provides, for the first time, estimates of the $9 \mathrm{yr}$ nutation terms. We emphasize that our results do not depend on any geophysical hypothesis, that they are unconstrained, and that they have been obtained with the full VLBI and LLR information matrices retained. The overall agreement between our estimates and the independent results of Herring (1988) and Zhu et al. (1990) is good.

\section{Acknowledgments}

We thank R. P. Branson for helpful discussions concerning the implementation of the SRIF algorithm. P. C. was supported in part by a Lavoisier Fellowship of the Ministère des Affaires Etrangères, France. This research was carried out by the Jet Propulsion Laboratory, California Institute of Technology, under a contract with NASA.

\section{References}

Bierman, G. J.: 1977, Factorization Methods for Discrete Sequential Estimation, Academic Press, New York.

Herring, T. A.: 1988, in BIH Annual Report for 1987, Paris, France, p. D-106.

Herring, T. A., Gwinn, C. R., and Shapiro, I. I.: 1986, J. Geophys. Res. 91, 4745.

International Earth Rotation Service: 1989, IERS Standards, IERS Technical

Note 3, Ed. D. D. McCarthy, Paris, France.

Sovers, O. J.: 1990, in IAU Symposium 141, Inertial Coordinate System on the Sky,

Eds. J. H. Lieske and V. Abalakin, Kluwer Academic Publishers, Dordrecht, p. 261.

Sovers, O. J. and Fanselow, J. L.: 1987, JPL/NASA Publ. 83-39, Rev. 3.

Williams, J. G., Newhall, X X, and Dickey, J. O.: 1990, Astron. Astrophys. (in press).

Zhu, S. Y., Groten, E., and Reigber, Ch.: 1990, Astron. J. 99, 1024. 